\title{
Analyse contrastive des changements syntaxiques et sémantiques affectant le discours direct dans The Pearl et La Perle de John Steinbeck
}

\section{Grégoire Lacaze}

\section{(2) OpenEdition \\ Journals \\ Édition électronique \\ URL : http://journals.openedition.org/esa/1126 \\ DOI : $10.4000 /$ esa. 1126 \\ ISSN : 2650-2623 \\ Éditeur \\ Société de stylistique anglaise}

Édition imprimée

Date de publication : 1 avril 2013

Pagination : 173-189

ISSN : 2116-1747

\section{Référence électronique}

Grégoire Lacaze, «Analyse contrastive des changements syntaxiques et sémantiques affectant le discours direct dans The Pearl et La Perle de John Steinbeck », Études de stylistique anglaise [En ligne], 5 | 2013, mis en ligne le 19 février 2019, consulté le 21 avril 2019. URL : http://

journals.openedition.org/esa/1126; DOI : 10.4000/esa.1126 


\title{
ANALYSE CONTRASTIVE DES CHANGEMENTS SYNTAXIQUES ET SÉMANTIQUES AFFECTANT LE DISCOURS DIRECT DANS THE PEARL ET LA PERLE DE JOHN STEINBECK
}

\author{
Grégoire Lacaze \\ Aix-Marseille Université - LERMA - EA 853
}

\begin{abstract}
This paper follows an initial publication (Lacaze 2012) devoted to a methodological reflection on the choices adopted in Gallimard's 1950 French translation of Steinbeck's The Pearl in relation to the introduction of direct speech. It aims at investigating certain syntactic and semantic changes emerging in utterances of direct speech in La perle. Changes such as a substitution of the verb of saying, a shift in the contextualizing sequence, the cancellation or the insertion of a contextualizing sequence are explored here in the original text and the French translation.
\end{abstract}

Keywords: introduction, direct speech, translation studies, contrastive approach, English, French, John Steinbeck, The Pearl, La perle, syntax, semantics, stylistics

\section{Introduction}

Notre première étude (Lacaze 2012) visant à mettre en évidence certains choix stylistiques adoptés par les traducteurs Marcel Duhamel et Renée Vavasseur dans La perle ${ }^{1}$, traduction française de The Pearl de John Steinbeck parue aux Éditions Gallimard en 1950, a tenté de montrer l'ampleur des défis

1 L'édition choisie est celle publiée aux Éditions Gallimard en 2000 dans laquelle la traduction se trouve toujours en regard du texte source. 
qu'un traducteur doit relever lorsqu'il entreprend la traduction d'une œuvre, en particulier pour traduire les occurrences de discours direct (noté DD).

Cette étude mettait en lumière divers phénomènes comme la variation de la composition du segment contextualisant (noté SC) sous l'influence du cotexte, l'inversion sujet-verbe de parole dans les incises ou encore les choix affectant la marque de ponctuation qui sépare le discours cité du SC qui l'introduit.

L'analyse proposée dans le présent article va se concentrer sur quelques bouleversements syntaxiques notables affectant l'introduction du discours direct en anglais et en français. Seront successivement étudiés le changement de verbe introducteur contenu dans le SC, le déplacement du SC relativement au discours cité lors de l'opération de traduction, l'effacement partiel ou total du SC entre ces deux textes, l'ajout d'un SC si ce dernier n'est pas présent dans le texte source et le changement de forme de discours rapporté (noté DR) au passage d'une langue à l'autre. Ces choix qui transforment profondément les structures syntaxiques seront envisagés à l'aune de considérations pragmatiques, sémantiques et stylistiques.

\section{Différences sémantiques impliquées par le changement de verbe introducteur}

Parmi les nombreux changements syntaxiques et sémantiques qui peuvent intervenir entre le texte source et sa traduction, le changement de sémantisme du verbe introducteur mérite d'être pris en considération.

La liberté prise par les traducteurs dans le choix du verbe introducteur français par rapport au sémantisme du verbe introducteur du texte source peut être la trace d'une stratégie énonciative privilégiée par les traducteurs. Parmi les différences existant entre l'anglais et le français, il est établi que l'on recommande souvent d'éviter la répétition d'un même terme en français. Pour se conformer à cet usage, les traducteurs sont donc censés changer fréquemment de verbe introducteur. Pour autant, le choix du sémantisme du verbe introducteur leur revient pleinement.

Traduction du verbe say par un verbe plus riche sémantiquement: mise en relief de l'acte illocutionnaire

Le verbe say est le verbe du report de paroles par excellence. La présence de ce verbe à mode fini comme verbe de parole dans un segment introducteur de DD permet l'attribution d'un dit à une origine énonciative tout en assurant les repérages prédicatifs et énonciatifs de l'acte rapporté. Le verbe 
dire, qui peut être envisagé comme l'homologue du verbe say en français, est souvent remplacé par d'autres verbes au sémantisme plus riche dans la traduction. Les deux énoncés suivants en sont une illustration :

"This is what the pearl will do," said Kino. (Steinbeck 84)

«Voilà ce que fera la perle », déclara Kino. (85)

The doctor's eyes watered in their little hammocks. "I will give him something to try to turn the poison aside," he said. (Steinbeck 96)

Les yeux du docteur larmoyèrent dans leurs petites poches. " Je vais lui donner quelque chose pour essayer de détourner le poison », annonça-t-il. (97)

Les verbes déclarer et annoncer sont un peu plus riches sémantiquement que le verbe dire. Ils portent en eux un certain degré de «force illocutionnaire $»^{2}$. Il arrive également que le verbe say soit traduit par le verbe faire, illustrant d'une certaine manière la performativité de l'acte énonciatif origine comme dans l'énoncé suivant :

The gate closed a little, and the servant refused to speak in the old language. "A little moment," he said. "I go to inform myself," and he closed the gate and slid the bolt home. (Steinbeck 44)

La porte se rabattit un peu, et le domestique refusa de répondre dans sa langue maternelle. «Un instant, fit-il, je vais me renseigner» et il referma la porte et mit le loquet. (45)

\section{Traduction du verbe say par un verbe impliquant une meilleure intégration d'une réplique dans le report d'un dialogue}

Parfois, le verbe say est remplacé par un verbe favorisant l'ancrage d'une réplique dans une temporalité afin de mettre en évidence l'enchaînement des répliques et la cohésion des échanges discursifs. L'effet de mise en mémoire explicite de la prise de parole successive des personnages se répercute sur la

2 L'expression force illocutionnaire, encore appelée force illocutoire, est dérivée de l'expression illocutionary force, introduite par John Langshaw Austin $(1975,146)$ : "With the performative utterance, we attend as much as possible to the illocutionary force of the utterance [...]». Voici la définition proposée par Dominique Maingueneau (1991,11-2) de l'expression force illocutoire : «Le langage n'a pas pour unique fonction de transmettre des contenus, il est aussi action. Tout énoncé, outre le contenu qu'il véhicule, accomplit un certain type d'acte de langage à l'égard de son allocutaire : actes de promettre, questionner, menacer, etc. On dit que les énoncés sont affectés d'une certaine force illocutoire (promesse, question...) qui peut être explicitée par la syntaxe (cf. JE TE JURE QU'il pleut où la force illocutoire est marquée par jurer, verbe dit "performatif" en ce sens que seule son énonciation par un je permet d'accomplir l'acte de jurer), ou par l'intonation seulement (d'où l'ambiguïté à l'écrit d'un énoncé comme il partira, qui peut constituer aussi bien une promesse qu'une menace, etc.), ou encore par le contexte. » (C'est l'auteur qui souligne.) 
composition des SC, notamment au niveau du choix des verbes introducteurs. Ainsi, le verbe say peut être remplacé par un verbe indiquant le positionnement de la réplique d'un locuteur origine par rapport aux répliques de son interlocuteur mais aussi par rapport aux autres répliques de ce locuteur origine :

“The doctor would not come," Kino said to Juana.

$[\ldots]$

"Then we will go to him," Juana said, and with one hand she arranged her dark-blue shawl over her head [...]. (Steinbeck 38)

« Le docteur ne voudra pas venir », dit-il à Juana.

$[\ldots]$

«Eh bien! nous irons chez lui », répondit Juana et, d'une main, elle drapa son châle bleu sombre autour de sa tête [...]. (39)

Le verbe répondre souligne ici la réaction de Juana à la prise de parole de Kino. Un phénomène similaire se retrouve dans l'énoncé suivant :

The doctor put his cup down gently before he let his anger rise.

"Have I nothing better to do than cure insect bites for 'little Indians'? I am a doctor, not a veterinary."

"Yes, Patron," said the servant. (Steinbeck 48)

Le docteur posa soigneusement sa tasse avant de donner libre cours à sa colère.

«Est-ce que je n'ai rien de mieux à faire que de soigner les piqûres d'insectes de "petits Indiens"? Je suis médecin, pas vétérinaire.

- Oui, Patron, répondit le domestique. [...]» (49)

L'emploi du verbe répondre comme verbe introducteur se justifie par la volonté des traducteurs d'inscrire la réplique du domestique dans le dialogue en réponse à la question posée par le docteur.

\section{Traduction du verbe say par un verbe facilitant l'interprétation du report de paroles par le lecteur}

Les traducteurs deviennent, d'une certaine manière, des auteurs lorsqu'ils mettent en œuvre leur activité de traduction. Ils sont donc pleinement responsables du choix des verbes introducteurs de DD. Parmi les degrés de liberté qui sont à leur disposition, il semble que les traducteurs de The Pearl aient choisi délibérément d' «accompagner » le lecteur au cours de sa lecture des dialogues notamment. Les traducteurs mettent ainsi en évidence les prises de parole respectives des participants à un acte énonciatif origine. Le verbe introducteur devient ce que nous appelons un «auxiliaire interprétatif $»^{3}$. Le

3 Le verbe introducteur peut être considéré comme un «facilitateur de l'interprétation », pour reprendre une expression employée par Anna Jaubert $(2005,8)$. 
sémantisme du verbe introducteur n'est plus aussi neutre que lorsque le verbe dire est employé. La remarque précédente s'inscrit dans l'hypothèse de travail formulée par Mona Baker et évoquée par Mathieu Guidère $(2010,95)$ : «"l'hypothèse de l'explicitation" faite par Baker(1997) selon laquelle les textes traduits sont en général plus explicites que les textes sources ».

Dans l'énoncé suivant déjà cité, le SC contient le verbe expliquer alors que le verbe say est le verbe introducteur dans le texte source :

The man who looked out at him was one of his own race. Kino spoke to him in the old language. "The little one - the first-born - has been poisoned by the scorpion," Kino said. "He requires the skill of the healer." (Steinbeck 44)

L'homme qui l'observait de l'intérieur était de sa race. Kino lui parla dans leur langue natale : «Le petit... notre premier-né... a été empoisonné par le scorpion, expliqua-t-il. Il a besoin de la science du guérisseur. » (45)

Une nouvelle fois, le verbe introducteur expliquer en français est plus riche sémantiquement que son homologue en anglais. Il tend à expliciter le contenu du discours cité et à légitimer la prise de parole de Kino, alors que dans le texte source, le verbe say assure seulement la mise en relation d'un dit avec une origine énonciative.

Par ailleurs, l'on peut noter dans l'incise en français l'ajout obligatoire du phonème $/ t /$, c'est-à-dire d'une «épenthèse $»^{4}$ pour des raisons d'aisance articulatoire.

Faciliter l'appropriation du texte par un lectorat francophone semble être une position souvent adoptée par les traducteurs de The Pearl. C'est donc une stratégie cibliste qui est préconisée. Certaines caractéristiques du texte source tendent à être quelque peu gommées, masquées par le choix d'une volonté affichée de rendre la lecture du texte en français aussi naturelle que possible en faisant disparaître des répétitions que l'on rencontre dans le texte origine, notamment la présence du verbe say dans les incises de DD.

\section{Recherche d'une diversité d'emploi des verbes introducteurs en français}

Il semble exister ici certaines similitudes entre le discours fictionnel et le discours journalistique où le verbe say est très souvent employé, comme le rappelle Jean-Claude Sergeant $(2011,37)$ qui relève « la présence hégémonique du verbe d'attribution de base to say». Selon lui, «le souci de diversification du lexique ne semble pas être l'une des contraintes imposées aux journalistes

4 L'épenthèse est définie ainsi : «Phénomène consistant dans l'apparition, à l'intérieur d'un mot ou groupe de mots, d'un phonème adventice d'origine ou de nature non étymologique qui contribue à en faciliter l'articulation » (Le Trésor de la Langue Française Informatisé). 
britanniques ». A contrario, la presse française semble avoir recours à une grande diversité de verbes introducteurs pour éviter la répétition du verbe dire : Jean-Claude Sergeant $(2011,37)$ suppose « une volonté des professionnels de l'écriture de presse de s'affranchir du carcan des formules standardisées ». Ces remarques qui portent sur des énoncés de discours journalistique semblent transposables à l'œuvre de fiction étudiée, à savoir, The Pearl, et à sa traduction, La perle. John Steinbeck utilise abondamment le verbe introducteur say tandis que les traducteurs français ont tendance à employer en alternance divers verbes introducteurs comme les verbes déclarer, faire et souffler pour n'en citer que quelques-uns.

L'énoncé suivant illustre l'emploi du verbe souffler comme verbe de parole :

"There is a way," he said.

"But they will kill you." (Steinbeck 218)

«Il y a un moyen, souffla-t-il.

- Mais ils vont te tuer ! [...]» (219)

Les traducteurs semblent ainsi s'efforcer de parcourir l'axe paradigmatique permettant de remplacer le verbe dire par un autre verbe plus riche sémantiquement. Ce faisant, la traduction est colorée d'un sémantisme qui n'était pas présent dans le texte source, ce qui tend à mettre en avant l'acte créatif des traducteurs.

\section{Bouleversements syntaxiques entre le texte source et le texte cible et conséquences sur le sémantisme}

L'opération de traduction peut s'accompagner de changements syntaxiques plus ou moins importants. Il arrive qu'un changement syntaxique puisse entraîner divers changements qui sont liés à ce bouleversement de la syntaxe d'une phrase. L'énoncé suivant contient plusieurs changements qui se combinent et illustrent la liberté dont jouissent les traducteurs malgré la présence de certaines contraintes :

\footnotetext{
And Juana, sitting by the fire hole, watched him with questioning eyes, and when he had buried his pearl she asked, "Who do you fear?" (Steinbeck 108)

Kino searched for a true answer, and at last he said, "Everyone." And he could feel a shell of hardness drawing over him. (Steinbeck 110)

Et Juana, assise près du foyer, le regarda faire d'un air interrogateur et, lorsqu'il eut enterré la perle, elle lui demanda : «Qui crains-tu donc ? » (109)

Kino chercha une réponse sincère et finalement avoua : "Tout le monde », et il sentit la dure carapace se tendre tout autour de lui. (111)
} 
Si l'on s'intéresse au SC introduisant les propos de Kino dans les deux extraits, le pronom personnel qui désigne le locuteur origine n'apparaît plus en français car les traducteurs ont adopté une structure syntaxique différente de celle du texte source. L'appellatif Kino est en facteur commun du verbe chercher et du verbe avouer. Par ailleurs, le verbe avouer n'a pas la seule fonction d'introduire un report de paroles; en tant que «verbe factif $»^{5}$, il présuppose la vérité du contenu propositionnel figurant dans le discours cité. Le choix de ce verbe par les traducteurs permet au lecteur d'anticiper la réaction de Kino avant que ses propos soient rapportés.

Le déplacement du SC constitue visuellement la transformation la plus évidente lors du passage du texte source au texte cible. En d'autres termes, la position du SC dans l'une des trois positions possibles (Lacaze 2011) n'est pas conservée dans la traduction en français. Ce déplacement mérite toute l'attention du linguiste-stylisticien car la perception de l'acte de parole par le lecteur s'en trouve irrémédiablement affectée.

Nous avons envisagé tout SC comme un des éléments structurels fondamentaux sur lesquels les traducteurs doivent se pencher. Il nous parait donc logique d'envisager la variation de la position du SC relativement au discours cité entre l'œuvre de Steinbeck et sa traduction en français.

\section{Variation de la position du SC entre le texte source et le texte cible}

Nous avons montré (Lacaze 2011,27) qu'il existe trois positions possibles pour un $\mathrm{SC}$ relativement au discours cité :

- la «position initiale »: le SC précède le discours cité ;

- la «position médiane»: le SC est encadré par deux fragments de discours cité ;

- la «position finale » : le SC suit le discours cité.

L'on constate que les traducteurs n'ont pas toujours choisi de respecter dans leur traduction la position du SC dans le texte source. Diverses combinaisons sont possibles et vont être présentées successivement.

La position occupée par le SC dans le texte source n'est pas nécessairement conservée dans le texte cible, les changements syntaxiques ayant une répercussion inévitable sur le sémantisme de l'énoncé. Un SC en position initiale dans le texte source peut être en position finale dans la traduction, comme dans l'énoncé suivant :

5 Le verbe avouer est classé par Catherine Kerbrat-Orecchioni $(1980,116)$ dans la catégorie des verbes factifs : il « est à la fois modalisateur intrinsèque (verbe factif-positif), et axiologique, puisqu'il dénonce comme une faute le contenu de l'aveu ». 
In the pearl he saw Juana and Coyotito and himself standing and kneeling at the high altar, and they were being married now that they could pay. He spoke softly: "We will be married - in the church." (Steinbeck 80)

Dans la perle, il vit Juana, Coyotito et lui-même, debout puis agenouillés devant le grand autel et le prêtre les mariait maintenant qu'ils avaient de quoi payer. "Nous allons nous marier... à l'église », annonça-t-il à voix basse. (81)

Dans l'énoncé source déjà étudié, l'acte de parole origine est envisagé comme un acte fondateur grâce au SC en position initiale. origine :

L'énoncé suivant met en jeu également la musicalité de l'acte énonciatif

He heard a murmur behind him. Juana was whispering, "Coyotito." (Steinbeck 216)

Il entendit un murmure dans son dos : «Coyotito », chuchota Juana. (217)

Le SC est rejeté en position finale dans la traduction. À nouveau, il semble exister une analogie entre ce discours fictionnel et le discours journalistique. Jean-Claude Sergeant $(2011,38)$ fait remarquer que « les journalistes français intègrent [les éléments de discours cités] dans une fluidité narrative qui vise à en gommer l'externalité ». Déplacer le SC en position finale dans un discours fictionnel tend à atténuer la rupture syntaxique entre récit et occurrence de DD.

C'est aussi le constat qu'établissent Sylvie Hanote et Hélène Chuquet $(2004,25)$ : lorsque le SC est en position médiane ou en position finale, «la construction d'un événement de parole dans le récit ne semble plus envisagée comme première mais comme parenthétique », le segment introducteur «ne faisant que qualifier [les propos cités au discours direct] comme étant les paroles d'un locuteur identifié et déterminé dans le récit ».

Le déplacement d'un SC de la position initiale dans le texte source vers la position médiane dans le texte cible amoindrit donc le statut de l'acte énonciatif origine, comme le rappellent Sylvie Hanote et Hélène Chuquet $(2004,25)$ : «lorsque [le SC] est placé avant le discours direct, la prise de parole est présentée comme un événement pris dans le récit, tandis que lorsque [le SC] est placé [en position médiane ou en position finale], le contenu de parole est simplement posé sans annonce ». Ceci est illustré par l'exemple suivant :

The priest said, "It is pleasant to see that your first thoughts are good thoughts. God bless you, my children." (Steinbeck 90)

«Il est doux de constater, poursuivit le prêtre, que vos premières pensées sont de bonnes pensées. Dieu vous bénisse, mes enfants. » (91) 
L'incise de DD en français s'insère comme une expression parenthétique au sein du discours cité. Contenant le verbe poursuivre, elle assure un repérage du tour de parole du prêtre par rapport à sa première prise de parole qui figure dans le cotexte gauche. Geneviève Salvan $(2005,137)$, dans son étude sur l'incise de DR dans le roman français, fait remarquer qu'une incise contenant le verbe poursuivre «implique une continuité textuelle». Cette incise «fonctionne dans un premier temps par rapport au contexte gauche [...]. Elle inscrit le fragment de discours direct dans la continuité du précédent ». Cette remarque est en accord avec la stratégie poursuivie par les traducteurs qui cherchent à favoriser une meilleure insertion de chaque réplique dans un dialogue.

De manière analogue, si un SC en position finale dans le texte source est déplacé en position initiale dans la traduction, l'acte de parole est alors davantage inscrit comme un événement dans le récit.

Soit l'énoncé suivant :

She paused, her hands helpless in front of her, and then, "Yes, my brother-in-law," she said. (Steinbeck 174)

Elle s'arrêta, les mains ballantes devant elle, puis elle répondit : « Bien, beau-frère. » (175)

Le changement de position s'accompagne ici dans la traduction du choix d'un verbe introducteur précisant l'enchaînement des tours de parole entre les participants à l'acte énonciatif.

Nous avons montré par ailleurs qu'un SC peut être constitué de plusieurs fragments encadrant le discours cité (Lacaze 2011,41). Parfois, seul un fragment du SC change de position, comme dans l'extrait suivant :

Kino could feel warm blood running down from his forehead, and he could hear Juana calling to him, "Kino! Kino!" And there was terror in her voice. (Steinbeck 112)

Kino sentait le sang chaud couler sur son front et entendit Juana l'appeler d'une voix étranglée de terreur : « Kino ! Kino ! » (113)

L'approche transphrastique peut permettre d'analyser les choix de traduction dans l'énoncé ci-dessus. La phrase indépendante And there was terror in her voice fait partie du SC qui signale le report de paroles au DD. Elle complète la première partie du SC contenant le segment textuel he could hear Juana calling to him qui précède les paroles rapportées. Les traducteurs sont alors amenés à créer un SC rassemblant toutes les informations contenues dans deux segments autonomes syntaxiquement en anglais. Le contenu sémantique du segment en position finale dans le texte source a été inséré dans le segment en position initiale dans la traduction sous la forme du syntagme prépositionnel d'une voix étranglée de terreur. Les deux fragments constituant le SC et séparés dans le texte source sont fusionnés dans le texte cible. Ainsi, même s'il 
existe une indépendance syntaxique entre le discours cité et le fragment de SC qui le suit dans le texte source, la subordination sémantique existe néanmoins et elle est mise en évidence par le changement syntaxique retenu par les traducteurs.

Un SC en position finale dans le texte source peut également apparaître en position médiane dans la traduction, comme l'atteste l'énoncé suivant :

At last he handed the baby back to Juana, and he turned to Kino. "I think the poison will attack within the hour," he said. (Steinbeck 98)

Finalement, il rendit le petit à Juana et se tourna vers Kino. «Je pense, dit-il, que le poison se manifestera dans l'heure qui vient. [...]» (99)

Dans son étude sur la traduction de certains prédicats subjectifs comme le verbe think, Bruno Poncharal $(2000,3)$ estime que «THINK peut avoir un double statut : il peut fonctionner comme une incise (parenthetical), indiquant explicitement au lecteur-co-énonciateur la source des pensées représentées ». Il peut aussi «prend[re] sa fonction de verbe d'opinion, c'est-à-dire, sa fonction modalisatrice ».

Avec le SC en position finale, le segment textuel I think du discours cité permet au locuteur origine de «modaliser son assertion» (Poncharal 2000, 4). La présence de ce segment textuel «explicite le point de vue subjectif par rapport auquel a lieu la validation » $(2000,5)$. Il semble envisageable de supprimer ce segment textuel dans le discours cité sans pour autant que le contenu sémantique du discours cité soit considérablement amoindri :

At last he handed the baby back to Juana, and he turned to Kino. "The poison will attack within the hour," he said.

L'énoncé modifié peut être considéré comme "plus assertif » en raison de l'absence du prédicat subjectif (Poncharal 2000, 5).

Le sens du contenu du discours cité va changer sous l'influence du déplacement du SC. Afin de mettre en évidence ce changement sémantique, l'on peut envisager le déplacement du SC dans le texte source afin qu'il occupe la position médiane :

At last he handed the baby back to Juana, and he turned to Kino. "I think, he said, the poison will attack within the hour."

Ce changement de position du SC modifie la réception du discours cité par le lecteur. Le fragment de discours cité I think est mis en valeur par détachement du reste de la phrase en raison de l'insertion de l'incise he said. Le verbe think doit alors être considéré comme un verbe d'opinion et l'implication du locuteur origine dans la validation de la proposition the poison will attack 
within the hour apparaît bien plus importante. La subjectivité du locuteur origine est mise en avant par l'insertion de l'incise en position médiane.

Cet effet est aussi présent dans la traduction proposée par les traducteurs où le verbe penser a la valeur d'un verbe d'opinion qui affirme le point de vue du locuteur origine.

À partir de l'étude comparative des énoncés présentés ci-dessus et de leur traduction, qui a pris en compte la variation de la position du SC, il a été mis en évidence que les traducteurs jouissent d'un degré de liberté certain puisqu'ils peuvent choisir de donner plus ou moins d'importance au report d'un acte énonciatif origine, suivant les stratégies narratives et énonciatives qu'ils privilégient.

\section{Suppression d'une incise avec ou sans effacement du SC dans la traduction}

Parmi les bouleversements syntaxiques affectant le SC, l'opération de traduction peut conduire à la suppression d'une incise de DD mais pas à l'effacement du SC. Pour autant, l'identification de l'origine énonciative ne pose pas nécessairement problème car l'environnement cotextuel peut compenser l'absence d'incise comme dans l'énoncé suivant :

And Kino saw Kino in the pearl [...]. His lips moved hesitantly over this - "A rifle," he said. "Perhaps a rifle." (Steinbeck 82)

Et Kino vit Kino dans la perle [...]. Ses lèvres hésitèrent avant de dessiner les mots : «Un fusil... un fusil, peut-être. » (83)

Alors que le segment His lips moved hesitantly over this décrit les mouvements des lèvres de Kino, le segment textuel Ses lèvres hésitèrent avant de dessiner les mots est orienté explicitement vers l'acte de parole, le syntagme nominal les mots étant en relation d'identification stricte avec le contenu propositionnel rapporté entre guillemets dans le cotexte droit. La disparition de l'incise he said dans le texte source est compensée par l'apparition du syntagme nominal les mots dans le texte cible. Au niveau stylistique, le choix retenu par les traducteurs fait naître une incertitude quant aux conditions de réalisation de l'acte de parole origine. Si les mots se dessinent sur les lèvres du locuteur origine, cela ne présuppose pas que ces paroles aient été prononcées à voix haute.

Par ailleurs, il arrive également qu'une réplique de DD ne soit pas introduite par un SC. Dans le texte source, un SC est présent grâce à l'incise he said tandis qu'il est effacé dans la traduction :

"What is it?" Juana asked.

"Hush", he said. (Steinbeck 192) 
Qu'y a-t-il ? demanda Juana.

- Chut! (193)

Malgré l'absence de SC dans la deuxième réplique de l'extrait de dialogue, comme deux personnages sont en présence dans cet échange énonciatif, le lecteur peut rétablir aisément l'attribution énonciative manquante, en raison de l'alternance des tours de parole ${ }^{6}$ entre les participants à l'acte énonciatif.

\section{Ajout d'une incise dans la traduction}

Le phénomène inverse du précédent peut se produire suite à l'opération de traduction, c'est-à-dire qu'une incise de DD fait son apparition dans la traduction alors que celle-ci est absente dans le texte source, comme dans l'énoncé suivant :

"[...] Boy," he called. And when his servant looked through the rear door, "Boy, go to such a one, and such another and such a third one. [...]" (Steinbeck 140)

« [...] Garçon!... » appela-t-il, et lorsque l'employé passa la tête par la porte du fond, il commanda : «Cours chez un tel, un tel et un tel. [...]» (141)

Alors que le texte source ne mentionne pas explicitement la source énonciative mais bien l'altérité interlocutive grâce au syntagme nominal his servant, les traducteurs ont choisi d'introduire une incise pour qu'il n'existe pas d'ambiguïté sur l'origine énonciative. C'est bien l'acheteur s'adressant à son employé qui est à l'origine des paroles rapportées entre guillemets. Toutefois, l'analyse du contenu du discours cité permet, en raison de la cohésion discursive, d'identifier la source énonciative car le SC he called est encadré par deux fragments de discours cité prononcés par le même locuteur origine.

\section{Changement de forme de DR entre le texte source et sa traduction}

Parmi les nombreux bouleversements syntaxiques recensés entre le texte source et sa traduction, un changement de forme de DR peut intervenir. Ainsi, une occurrence de DD peut être traduite par une occurrence de discours indirect classique (noté DIC).

Soit l'énoncé suivant :

"And mark this," they said. "Those dealers did not discuss these things. Each of the three knew the pearl was valueless."

6 L'alternance des tours de parole peut permettre de compenser l'absence de SC dans un dialogue. Ce phénomène d'effacement de l'attribution énonciative a été étudié dans notre article sur la nouvelle "Hills Like White Elephants" d'Ernest Hemingway (Lacaze 2010). 


\begin{abstract}
"But suppose they had arranged it before?"
"If that is so, then all of us have been cheated all of our lives."

Perhaps, some argued, perhaps it would have been better if Kino took the one thousand five hundred pesos. [...]

And now, said other fearful ones, now that he had defied them, those buyers will not want to deal with him at all. [...]

And other said, Kino is a brave man, and a fierce man; he is right. From his courage we may all profit. (Steinbeck 146)
\end{abstract}

Ce dialogue se caractérise par la présence de différentes sources énonciatives tout aussi faiblement déterminées les unes que les autres. Ces sources renvoient toutes aux villageois qui habitent à proximité de la maison de Kino. Les identités des individus composant les sources énonciatives demeurent inconnues. Seuls leurs propos sont rapportés dans un dialogue qui fait entendre une alternance de voix.

Il semble intéressant de remarquer que la première occurrence de DD est introduite par l'incise they said. Puis les deux répliques suivantes ne sont pas introduites. C'est le contenu des paroles qui est mis en avant et non pas l'identité de ces locuteurs. Dans les trois dernières répliques du dialogue, chaque discours cité est introduit par une incise. Toutefois, la mention d'une source énonciative faiblement déterminée coïncide avec la disparition des guillemets délimitant le discours cité. Il semble alors que le DD qui figure dans les trois dernières répliques du dialogue soit différent du DD présent dans les trois premières répliques. Monique De Mattia-Viviès $(2010,166)$ qualifierait ces répliques d'occurrence de «DD mimétique ». Selon elle, le DD mimétique se définit ainsi : un «DD sans guillemets et/ou sans marque de séparation entre l'incise et la citation [...] produisant un effet de discours en direct » (166).

Par ailleurs, chaque syntagme nominal apparaissant dans une des trois incises met en évidence la pluralité de chaque source énonciative. La «thèse autonymique »du discours direct, comme l'appelle Ulla Tuomarla $(1999,22)$ et qui a été mise en avant par Jacqueline Authier-Revuz (1992), est ici prise en défaut car il n'y a pas unicité du locuteur origine.

Les bouleversements énonciatifs et syntaxiques qui affectent ce polylogue ${ }^{7}$ représentent autant de contraintes dont les traducteurs doivent s'accommoder.

Voici les choix retenus par les traducteurs :

«Et remarquez-bien, disaient-ils, les acheteurs n'ont même pas discuté entre eux. Tous les trois, ils ont su que la perle était sans valeur.

- Oui, mais supposez qu'ils se soient mis d'accord à l'avance ?

- S'il en est ainsi, alors toute notre vie, nous aurions été volés.

- Peut-être, suggéra quelqu'un, peut-être aurait-il été préférable que Kino prît les quinze cents pesos. [...]»

7 Le terme polylogue est emprunté à Francis Berthelot $(2005,174)$. 
Et puis, disaient d'autres timorés, maintenant qu'ils les avaient défiés, les acheteurs n'accepteraient plus jamais de traiter avec lui. [...]

Mais d'autres répliquaient que Kino était un homme brave, un homme sans peur, qu'il avait raison et que son courage bénéficierait à tous. (147)

Le temps du verbe d'incise dans la première réplique du dialogue est l'imparfait et non pas le passé simple, ce qui semble être en accord avec la nature plurielle du sujet syntaxique. Les deuxième et troisième répliques ne sont pas introduites par un SC, de manière analogue au texte source. Par contre, alors qu'une rupture typographique et énonciative intervient avant la quatrième réplique dans le texte source, la traduction présente cette réplique comme faisant partie de l'échange discursif précédent. Cette réplique est précédée d'un tiret et est incluse dans les guillemets qui délimitent le dialogue. Par ailleurs, la source énonciative devient unique alors qu'elle était plurielle dans le texte source. Alors que la quatrième réplique était présentée dans le texte source comme ayant un statut similaire aux deux répliques suivantes ne contenant pas de guillemets, les traducteurs ont choisi de l'inclure dans le dialogue entre guillemets. De plus, la pluralité énonciative présente dans l'incise some said a disparu au profit d'une source énonciative unique désignée par quelqu'un et précédant le verbe introducteur suggérer au passé simple, ce qui semble en accord avec l'unicité de la prise de parole. Le contraste est évident avec la pluralité des sources énonciatives des deux répliques suivantes dans lesquelles les verbes introducteurs sont à l'imparfait. Les cinquième et sixième répliques ne sont plus signalées comme les occurrences d'un dialogue car les tirets et les guillemets sont absents. Les traducteurs ont ici changé profondément le style et le sens du texte source en traitant cette quatrième réplique de manière analogue aux trois premières répliques du dialogue.

Par ailleurs, les traducteurs ont aussi transformé la nature du dernier acte rapporté car la forme de DR a changé : ce report de paroles au DD dans le texte source se présente dans la traduction sous la forme d'une occurrence de DIC, ce qui implique un recalcul des repérages déictiques et temporels. Changer la forme de DR, c'est altérer la perception par le lecteur de l'acte origine. En effet, le texte source fait entendre et résonner les paroles origines ; il produit un effet de réel. Dans la traduction, le DIC tend à gommer la reproduction exacte de l'énonciation origine pour présenter seulement une reformulation ${ }^{8}$ des propos origines et l'investissement du locuteur rapporteur paraît bien plus important. De plus, au niveau du style, ce dernier report de paroles semble abandonner

8 Monique De Mattia-Viviès $(2010,152)$ présente ainsi les caractéristiques d'une occurrence de DIC : «De façon très schématique, le DIC (discours indirect classique) est censé rapporter un énoncé initial dans les termes du locuteur citant. Ce dernier doit théoriquement rester fidèle au contenu origine mais peut le reformuler et/ou le résumer, sélectionner les informations qu'il souhaite rapporter. Il est responsable des repérages temporels et des personnes (adaptation des repérages de l'énoncé origine à sa situation). » 
toute la fluidité du discours origine, notamment par la répétition du subordonnant que qui est mentionné trois fois.

\section{Conclusion}

Les traducteurs français de La perle dans l'édition de 1950 semblent avoir choisi délibérément de privilégier la réception de l'œuvre par un lectorat francophone. Certaines spécificités du texte source ont ainsi été gommées par certaines transformations syntaxiques et sémantiques qui affectent, à des degrés divers, le style de l'œuvre.

Les efforts conjugués des deux traducteurs offrent aux lecteurs francophones un accès aisé à l'œuvre de Steinbeck mais, parfois, le point de vue est sensiblement altéré, notamment lorsqu'un changement de forme de discours rapporté vient s'immiscer çà et là dans l'adaptation française.

Les modifications engendrées par la traduction en français de The Pearl affectent aussi bien le sémantisme du verbe introducteur que la syntaxe au moyen de l'ajout, du déplacement ou de la suppression d'un segment contextualisant. L'analyse de tous ces changements syntaxiques et sémantiques concourt à révéler l'influence des choix des traducteurs dans le processus de création que représente l'entreprise de traduction d'une œuvre.

Le «conflit» entre le «rythme» et le «signe» mis en avant par Henri Meschonnic $(2007,55)$ s'illustre bien dans la problématique de la traduction en français de The Pearl, les traducteurs ayant accordé la primauté à la fluidité et au rythme dans La perle. 


\section{Bibliographie}

\section{Corpus littéraire}

\section{A. Euvres de langue anglaise}

STEINBECK, John, (1947) 2000. The Pearl, Paris, Gallimard.

\section{B. Traduction}

Duhamel, Marcel et VAvasseur, Renée, (1950) 2000. Trad., La perle, Paris, Gallimard.

\section{Autres ouvrages cités}

Austin, John Langshaw, (1962) 1975. How to Do Things with Words: The William James Lectures delivered at Harvard University in 1955, J. O. Urmson and Marina Sbisà (ed), Harvard UP.

AuthIER-ReVuz, Jacqueline, 1992 (38-42). «Repères dans le champ du discours rapporté », L'information grammaticale 55.

BAKER, Mona, 1997 (175-186). "Corpus-based Translation Studies: the Challenges that Lie Ahead", Terminology, LSP and Translation: Studies in Language Engineering in Honour of Juan C. Sager, Harold Somers (ed), Amsterdam, John Benjamins Publishing.

Berthelot, Francis, 2005. Parole et dialogue dans le roman, Paris, Armand Colin.

De MatTIA-VIVIÈs, Monique, 2010 (151-180). «Du discours rapporté mimétique aux formes intrinsèquement hybrides », ANGLOPHONIA-SIGMA 28/2010, Toulouse, Presses Universitaires du Mirail.

«Épenthèse ». Le Trésor de la Langue Française Informatisé. 2 mai 2012 [http://atilf.atilf.fr/].

GUIDÈRE, Mathieu, (2008) 2010. Introduction à la traductologie - Penser la traduction : hier, aujourd'hui, demain, Bruxelles, De Boeck Université.

Hanote, Sylvie et Chuquet, Hélène, 2004. 'Who's speaking, please?' : le discours rapporté, Paris, Ophrys.

JAUBERT, Anna, 2005 (7-12). «Introduction. Cohésion et cohérence : étapes et relais pour l'interprétation », Cohésion et cohérence : études de linguistique textuelle, Anna Jaubert (éd), Lyon, ENS Éditions. 
Kerbrat-OrecCHIONI, Catherine, 1980. L'énonciation : de la subjectivité dans le langage, Paris, Armand Colin.

LACAZE, Grégoire, 2011 (25-44). «De l'incise au segment contextualisant: un changement d'horizon dans l'introduction du discours direct», Études de Stylistique Anglaise $\mathrm{N}^{\circ} 1$.

- - - 2012 (à paraître). «Regards croisés sur l'introduction du discours direct en anglais et en français : mise en contexte dans The Pearl et La perle de John Steinbeck », Études de Stylistique Anglaise $\mathrm{N}^{\circ} 3$.

- - , 2010. «Une analyse d'une production de discours rapporté : l'introduction des tours de parole dans la nouvelle "Hills Like White Elephants" d'Ernest Hemingway », E-rea 8.1. [http://erea.revues.org/pdf/1348]

MAINGUENEAU, Dominique, 1991. L'énonciation en linguistique française : embrayeurs, temps, discours rapporté, Paris, Hachette Supérieur.

MESCHONNIC, Henri, 2007. Éthique et politique du traduire, Lagrasse, Verdier, 2007.

PONCHARAL, Bruno, 2000 (1-31). «Problèmes de traduction posés par certains prédicats subjectifs au discours indirect libre », Linguistique contrastive et traduction 5 , Jacqueline Guillemin-Fischer (éd), Gap, Ophrys.

SALVAN, Geneviève, 2005 (113-144). «L'incise de discours rapporté dans le roman français du XVIII ${ }^{\mathrm{e}}$ au XX $\mathrm{XX}^{\mathrm{e}}$ siècle : contraintes syntaxiques et vocation textuelle », Cohésion et cohérence : études de linguistique textuelle, Anna Jaubert (éd), Collection Langages, Lyon, ENS Éditions.

SERGEANT, Jean-Claude, 2011. L'anglais du journalisme : comprendre et traduire, Paris, Ophrys.

TUOMARLA, Ulla, 1999. La citation mode d'emploi : sur le fonctionnement discursif du discours rapporté direct, Helsinki, Academia Scientiarum Fennica. 